\title{
General decay and blow-up of solutions for a nonlinear wave equation with memory and fractional boundary damping terms
}

\author{
Salah Boulaaras ${ }^{1}$, Fares Kamache ${ }^{2}$, Youcef Bouizem ${ }^{3}$, and Rafik Guefaifia ${ }^{2}$ \\ ${ }^{1}$ Qassim University \\ ${ }^{2}$ University of Tebessa \\ ${ }^{3}$ Universite des Sciences et de la Technologie d'Oran Mohamed Boudiaf
}

June 27, 2020

\begin{abstract}
The paper studies the global existence and general decay of solutions using Lyaponov functional for a nonlinear wave equation, taking into account the fractional derivative boundary condition and memory term. In addition, we establish the blow up of solutions with nonpositive initial energy.
\end{abstract}

\section{Hosted file}

GENERAL DECAY AND BLOW-UP.pdf available at https://authorea.com/users/337349/articles/462944general-decay-and-blow-up-of-solutions-for-a-nonlinear-wave-equation-with-memory-andfractional-boundary-damping-terms 